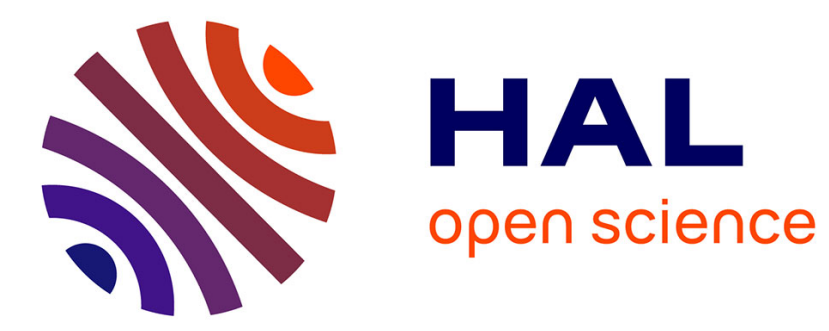

\title{
Transient responses of dynamical systems with random uncertainties
}

Christian Soize

\section{To cite this version:}

Christian Soize. Transient responses of dynamical systems with random uncertainties. Probabilistic Engineering Mechanics, 2001, 16 (4), pp.363-372. 10.1016/S0266-8920(01)00026-1 . hal-00686285

\section{HAL Id: hal-00686285 \\ https://hal.science/hal-00686285}

Submitted on 9 Apr 2012

HAL is a multi-disciplinary open access archive for the deposit and dissemination of scientific research documents, whether they are published or not. The documents may come from teaching and research institutions in France or abroad, or from public or private research centers.
L'archive ouverte pluridisciplinaire HAL, est destinée au dépôt et à la diffusion de documents scientifiques de niveau recherche, publiés ou non, émanant des établissements d'enseignement et de recherche français ou étrangers, des laboratoires publics ou privés. 


\title{
Transient Responses of Dynamical Systems with Random Uncertainties
}

\author{
C. Soize \\ Université de Marne-la-Vallée \\ Ing. 2000 Bat. Copernic, 5 Bd Descartes, 77454 Marne-la-Vallée Cedex 2, France \\ E-mail: soize@univ-mlv.fr
}

\begin{abstract}
A new approach is presented for modeling random uncertainties by a nonparametric model allowing transient responses of mechanical systems submitted to impulsive loads to be predicted in the context of linear structural dynamics. The probability model is deduced from the use of the entropy optimization principle whose available information is constituted of the algebraic properties related to the generalized mass, damping and stiffness matrices which have to be positive-definite symmetric matrices, and the knowledge of these matrices for the mean reduced matrix model. An explicit construction and representation of the probability model have been obtained and are very well suited to algebraic calculus and to Monte Carlo numerical simulation in order to compute the transient responses of structures submitted to impulsive loads. Finally, a simple example is presented.
\end{abstract}

Keywords: Random uncertainties; dynamical systems; structural dynamics; transient response; impulsive load; entropy optimization principle

\section{Introduction}

This paper deals with predicting the transient responses of structures submitted to impulsive loads in linear structural dynamics. In general, this kind of prediction is relatively difficult because the structural models have to be adapted to large, medium and small vibrational wavelengths which correspond to the low-, medium- and high-frequency ranges. Here, we are interested in the case where the impulsive load under consideration has an energy which is almost entirely distributed over a broad low-frequency band and for which prediction of the impulsive load response can be obtained with a reduced matrix model constructed using the generalized coordinates of the mode-superposition method associated with the structural modes corresponding to the $n$ lowest eigenfrequencies of the structure. Under the above 
assumptions and for a complex structure, dimension $n$ of the reduced matrix model generally has to be high (several dozen or hundred structural modes may be necessary to predict transient responses). However, it is known that the higher the eigenfrequency of a structural mode, the lower its accuracy because the uncertainties in the model increase (in linear structural dynamics and vibrations, the effects of uncertainties on the model increase with the frequency and it should be kept in mind that the mechanical model and the finite element model of a complex structure tend to be less reliable in predicting the higher structural modes). This is why random uncertainties in the mechanical model have to be taken into account. This is a fundamental problem in structural dynamics when the mechanical model has to be adapted to predict a transient response for which not only the low-frequency band is mainly concerned, but also the upper part of this low-frequency band and maybe the medium-frequency-band have to be taken into account. Random uncertainties in finite element models are usually modeled by using parametric models. Concerning such a parametric approach, for general developments, we refer the reader to Refs. [1-7] and for aspects related to stochastic finite elements, we refer the reader to Refs. [8-13]. The structural modes corresponding to the $n$ lowest eigenfrequencies of the complex structure are calculated using the finite element method (see for instance Refs. [14-18]). This paper presents a new nonparametric probabilistic model of random uncertainties for reduced matrix models of structures in order to predict transient responses due to impulsive loads. The information used does not require the description of the local parameters of the mechanical model. This nonparametric model of random uncertainties is based on a probability model introduced in Refs. [19,20] for symmetric positive-definite real random matrices deduced from the entropy optimization principle. The available information is only constituted of the mean value of the generalized mass, damping and stiffness matrices of the mean reduced matrix model which is deduced from the mean finite element model.

\section{A probability model for symmetric positive-definite random matrices}

In this section, we recall the main results established in Refs. [19,20] concerning the construction of a probability model for random matrices with values in the set $M_{n}^{+}(\mathbb{R})$ of all the $(n \times n)$ real symmetric positive-definite matrices using the entropy optimization principle which allows only the available information to be used. It should be noted that the results obtained and presented below differ from the known results concerning the Gaussian and circular ensembles for random matrices such as orthogonal (GOE), sympletic, unitary and antisymmetric hermitian ensembles which have been extensively studied in the literature (see for instance Ref. [21]). In addition, we complete the construction given in Refs. $[19,20]$ in order to obtain a consistent probabilistic model which allows the convergence properties to be 
studied when dimension $n$ approaches infinity.

2.1. Probability density function on the space of positive-definite symmetric real matrices and characteristic function

Let $M_{n}^{S}(\mathbb{R})$ be the set of all the $(n \times n)$ real symmetric matrices. Let $[\mathbf{A}]$ be a random matrix with values in $\mathbb{M}_{n}^{+}(\mathbb{R}) \subset \mathbb{M}_{n}^{S}(\mathbb{R})$ whose probability distribution

$$
P_{[\mathbf{A}]}=p_{[\mathbf{A}]}([A]) \widetilde{d} A
$$

is defined by a probability density function $[A] \mapsto p_{[\mathbf{A}]}([A])$ from $\mathbb{M}_{n}^{+}(\mathbb{R})$ into $\mathbb{R}^{+}=[0,+\infty[$ with respect to the measure (volume element) $\widetilde{d} A$ on $M_{n}^{S}(\mathbb{R})$ defined $[19,20]$ by

$$
\widetilde{d} A=2^{n(n-1) / 4} \Pi_{1 \leq i \leq j \leq n} d[A]_{i j}
$$

This probability density function is such that

$$
\int_{\mathbb{M}_{n}^{+}(\mathbb{R})} p_{[\mathbf{A}]}([A]) \widetilde{d} A=1
$$

For all $[\Theta]$ in $\mathbb{M}_{n}^{S}(\mathbb{R})$, the characteristic function of random matrix $[\mathbf{A}]$ with values in $\mathbb{M}_{n}^{+}(\mathbb{R}) \subset \mathbb{M}_{n}^{S}(\mathbb{R})$ is defined by $\Phi_{[\mathbf{A}]}([\Theta])=E\{\exp (i \ll[\Theta],[\mathbf{A}] \gg)\}$ in which $\ll[\Theta],[\mathbf{A}] \gg=\operatorname{tr}\left\{[\Theta][\mathbf{A}]^{T}\right\}=\operatorname{tr}\{[\Theta][\mathbf{A}]\}$ where tr denotes the trace of matrices and where $[\mathbf{A}]^{T}$ denotes the transpose of matrix $[\mathbf{A}]$. We then have

$$
\Phi_{[\mathbf{A}]}([\Theta])=\int_{\mathbb{M}_{n}^{+}(\mathbb{R})} \exp (i \ll[\Theta],[A] \gg) p_{[\mathbf{A}]}([A]) \widetilde{d} A
$$

\subsection{Available information for construction of the probability model}

We are interested in the construction of the probability distribution of a second-order random variable $[\mathbf{A}]$ with values in $\mathbb{M}_{n}^{+}(\mathbb{R})$ for which the available information is the mean value $[\underline{A}]$ of random matrix $[\mathbf{A}]$,

$$
E\{[\mathbf{A}]\}=\int_{\mathbb{M}_{n}^{+}(\mathbb{R})}[A] p_{[\mathbf{A}]}([A]) \widetilde{d} A=[\underline{A}],
$$

in which $E$ denotes the mathematical expectation and where mean value $[\underline{A}]$ is given in $\mathbb{M}_{n}^{+}(\mathbb{R})$. In addition, we assume that random matrix $[\mathbf{A}]$ is such that

$$
E\{\ln (\operatorname{det}[\mathbf{A}])\}=v \quad \text { with } \quad|v|<+\infty \quad .
$$


We proved in Refs. [19,20] that the constraint defined by Eq. (6) allows us to obtain the existence of moments related to the inverse random matrix $[\mathbf{A}]^{-1}$,

$$
E\left\{\left\|[\mathbf{A}]^{-1}\right\|_{F}^{\gamma}\right\}<+\infty \quad,
$$

in which $\gamma \geq 1$ is a positive integer and where $\|[A]\|_{F}=\left(\operatorname{tr}\left\{[A][A]^{T}\right\}\right)^{1 / 2}$ is the Frobenius norm of matrix $[A]$ in $M_{n}(\mathbb{R})$ (the set of all the $(n \times n)$ real matrices). Consequently, from Eqs. (3), (5) and (6), we deduce that the constraints imposed for the construction of the probability model of random matrix $[\mathbf{A}]$ with values in $M_{n}^{+}(\mathbb{R})$ are

$$
\begin{gathered}
\int_{\mathbb{M}_{n}^{+}(\mathbb{R})} p_{[\mathbf{A}]}([A]) \widetilde{d} A=1, \\
\int_{\mathbb{M}_{n}^{+}(\mathbb{R})}[A] p_{[\mathbf{A}]}([A]) \widetilde{d} A=[\underline{A}] \in \mathbb{M}_{n}^{+}(\mathbb{R}), \\
\int_{\mathbb{M}_{n}^{+}(\mathbb{R})} \ln (\operatorname{det}[\mathbf{A}]) p_{[\mathbf{A}]}([A]) \widetilde{d} A=v,
\end{gathered}
$$

in which $|v|<+\infty$.

\subsection{Probability model using the maximum entropy principle}

The measure of entropy [22] and the maximum entropy principle [23,24] are introduced to construct the probability model of random matrix $[\mathbf{A}]$ with values in $\mathbb{M}_{n}^{+}(\mathbb{R})$ based only on the use of the available information defined by Eqs. (8)-(10). Let $\lambda_{A}$ be the real parameter such that $1-\lambda_{A}$ is the Lagrange multiplier corresponding to the constraints defined by Eq. (10). It can then be proved $[19,20]$ that, for $\lambda_{A}>0$ and $[\Theta] \in \mathbb{M}_{n}^{S}(\mathbb{R})$, probability density function $p_{[\mathbf{A}]}([A])$ and characteristic function $\Phi_{[\mathbf{A}]}([\Theta])$ of positive-definite random matrix $[\mathbf{A}]$ are written as

$$
\begin{gathered}
p_{[\mathbf{A}]}([A])=\mathbb{1}_{\mathbb{M}_{n}^{+}(\mathbb{R})}([A]) \times c_{A} \times(\operatorname{det}[A])^{\lambda_{A}-1} \times \exp \left(-\frac{\left(n-1+2 \lambda_{A}\right)}{2} \operatorname{tr}\left\{[\underline{A}]^{-1}[A]\right\}\right), \\
\Phi_{[\mathbf{A}]}([\Theta])=\left\{\operatorname{det}\left(\left[I_{n}\right]-\frac{2 i}{\left(n-1+2 \lambda_{A}\right)}[\underline{A}][\Theta]\right)\right\}^{-\left(n-1+2 \lambda_{A}\right) / 2},
\end{gathered}
$$

in which det is the determinant of the matrices, $\left[I_{n}\right]$ is the $(n \times n)$ unity matrix and where $\mathbb{1}_{\mathbb{M}_{n}^{+}(\mathbb{R})}([A])$ is equal to 1 if $[A] \in \mathbb{M}_{n}^{+}(\mathbb{R})$ and is equal to zero if $[A] \notin M_{n}^{+}(\mathbb{R})$. When $\lambda_{A}$ is an integer, the probability distribution defined by Eq. (11) or (12) coincides with a Wishart distribution [25]. If $\lambda_{A}$ is not an integer, then the probability distribution defined by Eq. (11) or (12) is not a Wishart distribution. In Eq. (11), positive constant $c_{A}$ is written as

$$
c_{A}=\frac{(2 \pi)^{-n(n-1) / 4}\left(\frac{n-1+2 \lambda_{A}}{2}\right)^{n\left(n-1+2 \lambda_{A}\right) / 2}}{\left\{\Pi_{j=1}^{n} \Gamma\left(\frac{n-j+2 \lambda_{A}}{2}\right)\right\}(\operatorname{det}[\underline{A}])^{\left(n-1+2 \lambda_{A}\right) / 2}},
$$


where $\Gamma(z)$ is the gamma function defined for $\Re e z>0$ by $\Gamma(z)=\int_{0}^{+\infty} t^{z-1} e^{-t} d t$. The range of parameter $\lambda_{A}$ satisfying Eq. (7) in which $\gamma \geq 1$ is a positive integer has to be determined. For $\gamma \geq 1$, it can be proved that

$$
\lambda_{A}>\gamma+1 \Longrightarrow E\left\{\left\|[\mathbf{A}]^{-1}\right\|_{F}^{\gamma}\right\}<+\infty \quad, \quad \gamma \geq 1
$$

In addition, we have

$$
\lambda_{A}>0 \Longrightarrow E\left\{\|[\mathbf{A}]\|_{F}^{\eta}\right\}<+\infty \quad, \quad \forall \eta>0
$$

Equation (15) means that for $\lambda_{A}>0$, all the moments of random matrix $[\mathbf{A}]$ exist ( $\eta$ is any positive integer). The covariance $C_{j k, j^{\prime} k^{\prime}}^{A}=E\left\{\left([\mathbf{A}]_{j k}-[\underline{A}]_{j k}\right)\left([\mathbf{A}]_{j^{\prime} k^{\prime}}-[\underline{A}]_{j^{\prime} k^{\prime}}\right)\right\}$ of random variables $[\mathbf{A}]_{j k}$ and $[\mathbf{A}]_{j^{\prime} k^{\prime}}$ is written as

$$
C_{j k, j^{\prime} k^{\prime}}^{A}=\left(n-1+2 \lambda_{A}\right)^{-1}\left\{[\underline{A}]_{j^{\prime} k}[\underline{A}]_{j k^{\prime}}+[\underline{A}]_{j j^{\prime}}[\underline{A}]_{k k^{\prime}}\right\}
$$

and the variance $V_{j k}^{A}=C_{j k, j k}^{A}$ of random variable $[\mathbf{A}]_{j k}$ is such that $V_{j k}^{A}=\left(n-1+2 \lambda_{A}\right)^{-1}\left\{[\underline{A}]_{j k}^{2}+\right.$ $\left.[\underline{A}]_{j j}[\underline{A}]_{k k}\right\}$.

Since $[\underline{A}]$ is a positive-definite real matrix, there is an upper triangular matrix $\left[\underline{L}_{A}\right]$ in $M_{n}(\mathbb{R})$ such that

$$
[\underline{A}]=\left[\underline{L}_{A}\right]^{T}\left[\underline{L}_{A}\right],
$$

which corresponds to the Cholesky factorization of matrix $[\underline{A}]$. Considering Eq. (17), random matrix $[\mathbf{A}]$ can be written as

$$
[\mathbf{A}]=\left[\underline{L}_{A}\right]^{T}\left[\mathbf{G}_{A}\right]\left[\underline{L}_{A}\right],
$$

in which matrix $\left[\mathbf{G}_{A}\right]$ is a random variable with values in $\mathbb{M}_{n}^{+}(\mathbb{R})$. From Eqs. (5) and (18), we deduce that the mean value $\left[\underline{G}_{A}\right]$ of random matrix $\left[\mathbf{G}_{A}\right]$ is such that

$$
\left[\underline{G}_{A}\right]=E\left\{\left[\mathbf{G}_{A}\right]\right\}=\left[I_{n}\right]
$$

The probability density function $p_{\left[\mathbf{G}_{A}\right]}([G])$ with respect to measure $\widetilde{d} G$ on $\mathbb{M}_{n}^{S}(\mathbb{R})$ of random matrix $\left[\mathbf{G}_{A}\right]$ with values in $\mathbb{M}_{n}^{+}(\mathbb{R})$ is given by Eqs. (11) and (13) in which $[\underline{A}]$ has to be replaced by $\left[I_{n}\right]$. We then have

$$
p_{\left[\mathbf{G}_{A}\right]}([G])=\mathbb{1}_{\mathbb{M}_{n}^{+}(\mathbb{R})}([G]) \times C_{G_{A}} \times(\operatorname{det}[G])^{\lambda_{A}-1} \times \exp \left(-\frac{\left(n-1+2 \lambda_{A}\right)}{2} \operatorname{tr}[G]\right),
$$

in which positive constant $C_{G_{A}}$ is such that

$$
C_{G_{A}}=\frac{(2 \pi)^{-n(n-1) / 4}\left(\frac{n-1+2 \lambda_{A}}{2}\right)^{n\left(n-1+2 \lambda_{A}\right) / 2}}{\left\{\Pi_{j=1}^{n} \Gamma\left(\frac{n-j+2 \lambda_{A}}{2}\right)\right\}} .
$$


From Eqs. (16) and (18), we deduce that the covariance $C_{j k, j^{\prime} k^{\prime}}^{G}$ of random variables $\left[\mathbf{G}_{A}\right]_{j k}$ and $\left[\mathbf{G}_{A}\right]_{j^{\prime} k^{\prime}}$, defined by $C_{j k, j^{\prime} k^{\prime}}^{G}=E\left\{\left(\left[\mathbf{G}_{A}\right]_{j k}-\left[\underline{G}_{A}\right]_{j k}\right)\left(\left[\mathbf{G}_{A}\right]_{j^{\prime} k^{\prime}}-\left[\underline{G}_{A}\right]_{j^{\prime} k^{\prime}}\right)\right\}$, is written as

$$
C_{j k, j^{\prime} k^{\prime}}^{G}=\left(n-1+2 \lambda_{A}\right)^{-1}\left\{\left[\underline{G}_{A}\right]_{j^{\prime} k}\left[\underline{G}_{A}\right]_{j k^{\prime}}+\left[\underline{G}_{A}\right]_{j j^{\prime}}\left[\underline{G}_{A}\right]_{k k^{\prime}}\right\} .
$$

Since $\left[\underline{G}_{A}\right]=\left[I_{n}\right]$, the variance $V_{j k}^{G}=C_{j k, j k}^{G}$ of random variable $\left[\mathbf{G}_{A}\right]_{j k}$ is such that

$$
V_{j k}^{G}=\left(n-1+2 \lambda_{A}\right)^{-1}\left(1+\delta_{j k}\right),
$$

in which $\delta_{j k}=0$ if $j \neq k$ and $\delta_{j j}=1$. Let $\delta_{A}>0$ be defined by

$$
\delta_{A}=\left\{\frac{E\left\{\left\|\left[\mathbf{G}_{A}\right]-\left[\underline{G}_{A}\right]\right\|_{F}^{2}\right\}}{\left\|\left[\underline{G}_{A}\right]\right\|_{F}^{2}}\right\}^{1 / 2} .
$$

Equation (23) yields $E\left\{\left\|\left[\mathbf{G}_{A}\right]-\left[\underline{G}_{A}\right]\right\|_{F}^{2}\right\}=\sum_{j} \sum_{k} V_{j k}^{G}=n(n+1)\left(n-1+2 \lambda_{A}\right)^{-1}$ and since $\left\|\left[\underline{G}_{A}\right]\right\|_{F}^{2}=\left\|\left[I_{n}\right]\right\|_{F}^{2}=n$, we deduce that

$$
\delta_{A}=\left\{\frac{n+1}{n-1+2 \lambda_{A}}\right\}^{1 / 2},
$$

and consequently,

$$
\lambda_{A}=\ell_{A}(n)
$$

in which $n \mapsto \ell_{A}(n)$ is the mapping defined on the set $\mathbb{N}^{*}$ of all positive integers such that

$$
\ell_{A}(n)=\frac{1-\delta_{A}^{2}}{2 \delta_{A}^{2}} n+\frac{1+\delta_{A}^{2}}{2 \delta_{A}^{2}} .
$$

From Eqs. (14) and (25), we deduce that parameter $\delta_{A}$ has to be such that

$$
0<\delta_{A}<\sqrt{\frac{n+1}{n+1+2 \gamma}}<1 \quad, \quad \gamma \geq 1 \quad, \quad \forall n \geq 1 .
$$

Equation (28) shows that $\gamma$ has to be chosen as small as possible in order to increase the domain of possible values for $\delta_{A}$. From convergence considerations when $n \rightarrow+\infty$ and from Eq. (28), it can be deduced that $\gamma=2$ is an optimal value (see Ref. [26]). Let $n_{0} \geq 1$ be a fixed integer. Taking the value $\gamma=2$, we then deduce that, if parameter $\delta_{A}$ satisfies

$$
0<\delta_{A}<\sqrt{\frac{n_{0}+1}{n_{0}+5}},
$$

then, $\forall n \geq n_{0}$, we have $\lambda_{A}=\ell_{A}(n)>\gamma+1=3$ and consequently, Eq. (14) holds. These equations will be used as follows. The lower bound $n_{0}$ of positive integer $n$ is fixed. Then, the dispersion of the probability model is fixed by giving parameter $\delta_{A}$, independent of $n$, a value such that Eq. (29) is satisfied. For each value of integer $n \geq n_{0}$, parameter $\lambda_{A}=\ell_{A}(n)$ is then calculated by using Eq. (27). Consequently, $\lambda_{A}=\ell_{A}(n)$ appears as a function of $n$. 


\subsection{Monte Carlo simulation of random matrix $[\mathbf{A}]$ when $\lambda_{A}$ is an integer}

When $\lambda_{A}=\ell_{A}(n)$ is a positive integer, we introduce the positive integer $m_{A}$ such that

$$
m_{A}(n)=n-1+2 \ell_{A}(n) .
$$

Substituting Eq. (27) in the right-hand side of Eq. (30) yields

$$
m_{A}(n)=(n+1) / \delta_{A}^{2}
$$

Since $m_{A}(n)$ is a positive integer, it can be verified that the probability distribution defined by Eq. (11) or (12) is a Wishart distribution [25] and that random matrix $[\mathbf{A}]$ can be written $[19,20]$ as

$$
[\mathbf{A}]=\frac{1}{m_{A}(n)} \sum_{j=1}^{m_{A}(n)}\left(\left[\underline{L}_{A}\right]^{T} \mathbf{X}_{j}\right)\left(\left[\underline{L}_{A}\right]^{T} \mathbf{X}_{j}\right)^{T}
$$

in which $\left[\underline{L}_{A}\right]$ is the upper triangular matrix defined by Eq. (17) and where $\mathbf{X}_{1}, \ldots, \mathbf{X}_{m_{A}(n)}$ are independent random vectors, each vector $\mathbf{X}_{j}$ being an $\mathbb{R}^{n}$-valued second-order Gaussian random variable, centered and whose covariance matrix is $\left[C \mathbf{X}_{j}\right]=E\left\{\mathbf{X}_{j} \mathbf{X}_{j}^{T}\right\}=\left[I_{n}\right]$. Consequently, Eq. (32) gives an efficient procedure for the Monte Carlo simulation of random matrix $[\mathbf{A}]$.

\subsection{Monte Carlo simulation of random matrix $[\mathbf{A}]$ when $\lambda_{A}$ is a not an integer}

Let us now assume that $\lambda_{A}=\ell_{A}(n)$, given by Eq. (27), is a positive real number (the particular case for which $\lambda_{A}$ is a positive integer is presented above in Section 2.4). Since $\left[\mathbf{G}_{A}\right]$ defined by Eq. (18), is a random matrix with values in $\mathbb{M}_{n}^{+}(\mathbb{R})$, the Cholesky factorization allows us to write

$$
\left[\mathbf{G}_{A}\right]=\left[\mathbf{L}_{A}\right]^{T}\left[\mathbf{L}_{A}\right] \quad \text { a.s. }
$$

in which $\left[\mathbf{L}_{A}\right]$ is an upper triangular random matrix with values in $M_{n}(\mathbb{R})$. The following results, which allow a procedure for the Monte Carlo simulation of random matrix $[\mathbf{A}]$ to be defined, are proved $[19,20]$ : (1) Random variables $\left\{\left[\mathbf{L}_{A}\right]_{j j^{\prime}}, j \leq j^{\prime}\right\}$ are independent.

(2) For $j<j^{\prime}$, real-valued random variable $\left[\mathbf{L}_{A}\right]_{j j^{\prime}}$ can be written as $\left[\mathbf{L}_{A}\right]_{j j^{\prime}}=2^{-1 / 2}\left[\tilde{\mathbf{L}}_{A}\right]_{j j^{\prime}}$ in which $\left[\widetilde{\mathbf{L}}_{A}\right]_{j j^{\prime}}$ is a real-valued Gaussian random variable with zero mean and variance given by

$$
v=2\left(n-1+2 \ell_{A}(n)\right)^{-1} .
$$


(3) For $j=j^{\prime}$, positive-valued random variable $\left[\mathbf{L}_{A}\right]_{j j}$ can be written as $\left[\mathbf{L}_{A}\right]_{j j}=\sqrt{v \mathbf{Y}_{j}}$ in which $v$ is given by Eq. (34) and where $\mathbf{Y}_{j}$ is a positive-valued gamma random variable whose probability density function with respect to $d y$ is given by

$$
\Gamma_{j}(y)=\frac{\mathbb{1}_{[0,+\infty[}(y)}{\Gamma\left(\frac{n-j+2 \ell_{A}(n)}{2}\right)} y^{\frac{\left(n-j+2 \ell_{A}(n)\right)}{2}-1} e^{-y} .
$$

(4) We have $\left[\mathbf{G}_{A}\right]=\left[\mathbf{L}_{A}\right]^{T}\left[\mathbf{L}_{A}\right]$ and $[\mathbf{A}]=\left[\underline{L}_{A}\right]^{T}\left[\mathbf{G}_{A}\right]\left[\underline{L}_{A}\right]$.

\subsection{Probability model of a set of positive-definite symmetric real random matrices}

Let us consider $\nu$ random matrices $\left[\mathbf{A}_{1}\right], \ldots,\left[\mathbf{A}_{\nu}\right]$ with values in $M_{n}^{+}(\mathbb{R})$ such that for each $j$ in $\{1, \ldots, \nu\}$, the probability density function of random matrix $\left[\mathbf{A}_{j}\right]$ satisfies Eqs. (8)-(10). This means that only the mean values of the random matrices are known. Applying the maximum entropy principle, it can be proved that the probability density function $\left(\left[A_{1}\right], \ldots,\left[A_{\nu}\right]\right) \mapsto p_{\left[\mathbf{A}_{1}\right], \ldots,\left[\mathbf{A}_{\nu}\right]}\left(\left[A_{1}\right], \ldots,\left[A_{\nu}\right]\right)$ from $M_{n}^{+}(\mathbb{R}) \times \ldots \times \mathbb{M}_{n}^{+}(\mathbb{R})$ into $\mathbb{R}^{+}$with respect to the measure (volume element) $\widetilde{d} A_{1} \times \ldots \times \widetilde{d} A_{\nu}$ on $\mathbb{M}_{n}^{S}(\mathbb{R}) \times \ldots \times \mathbb{M}_{n}^{S}(\mathbb{R})$ is written as

$$
p_{\left[\mathbf{A}_{1}\right], \ldots,\left[\mathbf{A}_{\nu}\right]}\left(\left[A_{1}\right], \ldots,\left[A_{\nu}\right]\right)=p_{\left[\mathbf{A}_{1}\right]}\left(\left[A_{1}\right]\right) \times \ldots \times p_{\left[\mathbf{A}_{\nu}\right]}\left(\left[A_{\nu}\right]\right)
$$

which means that $\left[\mathbf{A}_{1}\right], \ldots,\left[\mathbf{A}_{\nu}\right]$ are independent random matrices.

\section{Transient responses of structural dynamical systems with random uncertainties}

\subsection{Introduction of the mean finite element model for transient responses to impulsive loads}

Let us consider the linear transient reponse of a damped fixed structure around a static equilibrium configuration considered as a natural state without prestresses, submitted to an impulsive load. We introduce the finite element model considered as the "mean finite element model" of this mechanical system. The transient response $\{\underline{\mathbf{y}}(t), t \geq 0\}$ of this mean finite element model is the solution of the following time evolution problem

$$
[\underline{\mathbb{M}}] \underline{\mathbf{y}}(t)+[\underline{\mathbb{D}}] \underline{\mathbf{y}}(t)+[\underline{\mathbb{K}}] \underline{\mathbf{y}}(t)=\mathbf{x}(t) \quad, \quad t \geq 0
$$

with the initial conditions,

$$
\underline{\mathbf{y}}(0)=\mathbf{y}_{0} \quad, \quad \underline{\mathbf{y}}(0)=\mathbf{y}_{1} \quad,
$$

in which $\underline{\mathbf{y}}=\left(\underline{y}_{1}, \ldots, \underline{y}_{m}\right)$ is the transient response vector of the $m$ DOFs (displacements and/or rotations), $\mathbf{x}=\left(x_{1}, \ldots, x_{m}\right)$ is the impulsive load vector of the $m$ inputs (forces and/or moments), $\mathbf{y}_{0}$ 
and $\mathbf{y}_{1}$ are the given initial conditions for displacement and velocity vectors $\underline{\mathbf{y}}$ and $\underline{\mathbf{y}}$ respectively. The mass, damping and stiffness matrices $[\underline{\mathbb{M}}],[\underline{\mathbb{D}}]$ and $[\underline{\mathbb{K}}]$ are positive-definite symmetric $(m \times m)$ real matrices (the structure being assumed to be fixed, there are no rigid body displacements). Note that underlined quantities refer to the "mean finite element model".

\subsection{Introduction of the mean reduced matrix model}

The mean reduced matrix model is constructed using the mode-superposition method. We consider the generalized eigenvalue problem $[\underline{K}] \underline{\varphi}=\underline{\lambda}[\underline{M}] \underline{\varphi}$ associated with the mean finite element model. Since $[\underline{\mathbb{K}}]$ is a positive-definite matrix, we have $0<\underline{\lambda}_{1} \leq \underline{\lambda}_{2} \leq \ldots$ and the associated eigenvectors $\left\{\underline{\varphi}_{1}, \underline{\varphi}_{2}, \ldots\right\}$ are such that $<[\underline{\mathbb{M}}] \underline{\varphi}_{\alpha}, \underline{\varphi}_{\beta}>=\underline{\mu}_{\alpha} \delta_{\alpha \beta}$ and $<[\underline{\mathbb{K}}] \underline{\varphi}_{\alpha}, \underline{\varphi}_{\beta}>=\underline{\mu}_{\alpha} \underline{\omega}_{\alpha}^{2} \delta_{\alpha \beta}$, in which $\underline{\omega}_{\alpha}=$ $\sqrt{\underline{\lambda}_{\alpha}}$ is the eigenfrequency of structural mode $\underline{\varphi}_{\alpha}$ whose normalization is defined by the generalized mass $\underline{\mu}_{\alpha}$ and where $<\mathbf{y}, \mathbf{x}>=y_{1} x_{1}+\ldots+y_{m} x_{m}$.

The mean reduced matrix model related to structural modes $\left\{\underline{\varphi}_{1}, \ldots, \underline{\varphi}_{n}\right\}$ with $n \ll m$ is then written as

$$
\underline{\mathbf{y}}_{n}(t)=[\underline{\Phi}] \underline{\mathbf{q}}(t)=\sum_{\alpha=1}^{n} \underline{q}_{\alpha}(t) \underline{\varphi}_{\alpha} \quad,
$$

in which $[\underline{\Phi}]$ is the $(m \times n)$ real matrix whose columns are constituted of structural modes $\left\{\underline{\varphi}_{1}, \ldots, \underline{\varphi}_{n}\right\}$ and where $\underline{\mathbf{q}}(t)=\left(\underline{q}_{1}(t), \ldots, \underline{q}_{n}(t)\right)$ is an $n$ real vector of the generalized coordinates which is the unique solution of the time evolution mean reduced matrix problem,

$$
[\underline{M}] \underline{\ddot{\mathbf{q}}}(t)+[\underline{D}] \underline{\dot{\mathbf{q}}}(t)+[\underline{K}] \underline{\mathbf{q}}(t)=\mathbf{F}(t) \quad, \quad t \geq 0
$$

with the initial conditions,

$$
\underline{\mathbf{q}}(0)=\mathbf{q}_{0} \quad, \quad \underline{\dot{\mathbf{q}}}(0)=\mathbf{q}_{1},
$$

in which the generalized force $\mathbf{F}(t)$ is an $n$ real vector such that

$$
\mathbf{F}(t)=[\underline{\Phi}]^{T} \mathbf{x}(t)
$$

The generalized mass, damping and stiffness matrices $[\underline{M}],[\underline{D}]$ and $[\underline{K}]$ are positive-definite symmetric $(n \times n)$ real matrices such that $[\underline{M}]_{\alpha \beta}=\underline{\mu}_{\alpha} \delta_{\alpha \beta},[\underline{D}]_{\alpha \beta}=<[\underline{\mathbb{D}}] \underline{\varphi}_{\beta}, \underline{\varphi}_{\alpha}>$ and $[\underline{K}]_{\alpha \beta}=\underline{\mu}_{\alpha} \underline{\omega}_{\alpha}^{2} \delta_{\alpha \beta}$. The initial conditions $\mathbf{q}_{0}$ and $\mathbf{q}_{1}$ are such that $\mathbf{q}_{0}=[\underline{M}]^{-1}[\underline{\Phi}]^{T}[\underline{\mathbb{M}}] \mathbf{y}_{0}$ and $\mathbf{q}_{1}=[\underline{M}]^{-1}[\underline{\Phi}]^{T}[\underline{M}] \mathbf{y}_{1}$. It is assumed that $n$ is such that $\underline{\mathbf{y}}_{n}(t)$, given by the mean reduced matrix model defined by Eqs. (39)-(42), is a good approximation of $\underline{\mathbf{y}}(t)$ given by the mean finite element model defined by Eqs. (37)-(38). 
The response ratio $\underline{r}_{n}(t)$ at time $t$, associated with the elastic energy of transient response $\underline{\mathbf{y}}_{n}(t)$ of the mean model and calculated with the mean reduced matrix model, is defined by

$$
\begin{aligned}
\underline{r}_{n}(t) & =<[\underline{\mathbb{K}}] \underline{\mathbf{y}}(t), \underline{\mathbf{y}}(t)>^{1 / 2}<[\underline{\mathbb{K}}] \underline{\mathbf{y}}_{S}, \underline{\mathbf{y}}_{S}>^{-1 / 2} \\
& =<[\underline{K}] \underline{\mathbf{q}}(t), \underline{\mathbf{q}}(t)>^{1 / 2}<\left[\underline{\mathbb{K}} \underline{\mathbf{y}}_{S}, \underline{\mathbf{y}}_{S}>^{-1 / 2},\right.
\end{aligned}
$$

in which $\underline{\mathbf{y}}_{S}=[\underline{\mathbb{K}}]^{-1} \mathbf{x}_{\max }$ with $\mathbf{x}_{\max }=\max _{t \geq 0} \mathbf{x}(t)$, corresponds to the maximum of the quasi-static response constructed with the mean finite elemnt model. The dynamic magnification factor $\underline{b}_{n}$ of the mean model (associated with the elastic energy) is defined by

$$
\underline{b}_{n}=\max _{t \geq 0} \underline{r}_{n}(t)
$$

For $n=m, \underline{r}_{m}(t)$ is the response ratio associated with transient response $\{\underline{\mathbf{y}}(t), t \geq 0\}$ of the mean finite element model (see Eqs. (37)-(38) and is written as

$$
\underline{r}_{m}(t)=<[\underline{\mathbb{K}}] \underline{\mathbf{y}}(t), \underline{\mathbf{y}}(t)>^{1 / 2}<[\underline{\mathbb{K}}] \underline{\mathbf{y}}_{S}, \underline{\mathbf{y}}_{S}>^{-1 / 2}
$$

The convergence with respect to $n$ can be analyzed in studying response ratio $\underline{r}_{n}(t)$ at time $t$ and dynamic magnification factor $\underline{b}_{n}$.

\subsection{Construction of a nonparametric model of random uncertainties for the reduced matrix model}

In this section, we introduce the principle of construction of a nonparametric model of random uncertainties, the available information being constituted of the mean reduced matrix model of the structure. It should be noted that the mean finite element model defined by Eqs. (37)-(38) is not able to predict the transient response due to impulsive load whose energy is distributed over a very broad frequency band, i.e. over the low-, medium- and high-frequency ranges (for instance, if there is energy in the medium-frequency range, more advanced probabilistic mechanical models such as the fuzzy structure theory have to be used to take into account the role played by the structural complexity [14]); the most that this kind of deterministic mean finite element model is able to predict is the transient response due to impulsive loads whose energy is mainly distributed over a broad low-frequency range for which the mean reduced matrix model defined by Eqs. (39)-(42) is suitable and allows the transient response to be predicted with good accuracy. This means that the mean finite element model does not constitute available information for constructing the nonparametric model of random uncertainties. However, the mean reduced matrix model defined by Eqs. (39)-(42) (with $n$ not too large) does constitute the available 
information for constructing the transient response of the mean model, then constructing the probability model of random uncertainties. This probabilistic model is a nonparametric model of random uncertainties because the sources of random uncertainties in the mechanical model which are due to uncertain mechanical parameters such as geometrical parameters, boundary conditions, junction stiffness, mass density, Young's modulus, etc., are not directly modeled by random variables or stochastic fields. These random uncertain geometrical and mechanical parameters mean that the generalized mass, damping and stiffness matrices of the reduced matrix model are random matrices. The nonparametric model of random uncertainties which is proposed consists in introducing a direct construction of a probabilistic model of these random generalized matrices. This random reduced matrix model associated with the mean reduced matrix model is then written as

$$
\mathbf{Y}_{n}(t)=[\underline{\Phi}] \mathbf{Q}(t)=\sum_{\alpha=1}^{n} Q_{\alpha}(t) \underline{\varphi}_{\alpha}
$$

in which $\mathbf{Q}(t)=\left(Q_{1}(t), \ldots, Q_{n}(t)\right)$ and where $\{\mathbf{Q}(t), t \geq 0\}$ is an $\mathbb{R}^{n}$-valued stochatic process such that

$$
\begin{gathered}
{[\mathbf{M}] \ddot{\mathbf{Q}}(t)+[\mathbf{D}] \dot{\mathbf{Q}}(t)+[\mathbf{K}] \mathbf{Q}(t)=\mathbf{F}(t), t \geq 0,} \\
\mathbf{Q}(0)=\mathbf{q}_{0} \quad, \quad \dot{\mathbf{Q}}(0)=\mathbf{q}_{1},
\end{gathered}
$$

in which generalized force $\mathbf{F}(t)$ is the $\mathbb{R}^{n}$-valued vector defined by Eq. (42), where initial conditions $\mathbf{q}_{0}$ and $\mathbf{q}_{1}$ have been previously defined and where $[\mathbf{M}],[\mathbf{D}]$ and $[\mathbf{K}]$ are the random generalized mass, damping and stiffness matrices with values in space $\mathbb{M}_{n}^{+}(\mathbb{R})$. The basic available information is the mean reduced matrix model which is constituted of mean generalized mass, damping and stiffness matrices $[\underline{M}],[\underline{D}]$ and $[\underline{K}]$ defined in Section 3.2 and which belong to $M_{n}^{+}(\mathbb{R})$. Random generalized mass, damping and stiffness matrices $[\mathbf{M}],[\mathbf{D}]$ and $[\mathbf{K}]$ are second-order random variables with values in $M_{n}^{+}(\mathbb{R})$ such that

$$
E\{[\mathbf{M}]\}=[\underline{M}] \quad, \quad E\{[\mathbf{D}]\}=[\underline{D}] \quad, \quad E\{[\mathbf{K}]\}=[\underline{K}] .
$$

In addition, in order to obtain a consistent probabilistic model and in particular, to obtain convergence properties of stochastic transient response $\left\{\mathbf{Y}_{n}(t), t \geq 0\right\}$ when dimension $n$ approaches infinity, we need to introduce information relative to the existence of moments of random variables $[\mathbf{M}]^{-1},[\mathbf{D}]^{-1}$ and $[\mathbf{K}]^{-1}$ (such as second-order moments). It should be noted that since random matrices $[\mathbf{M}],[\mathbf{D}]$ and $[\mathbf{K}]$ are almost surely positive definite, the inverse matrices exist almost surely, but the existence of second-order moments does not follow. We therefore introduce the following constraints,

$$
E\left\{\left\|[\mathbf{M}]^{-1}\right\|_{F}^{2}\right\}<+\infty \quad, \quad E\left\{\left\|[\mathbf{D}]^{-1}\right\|_{F}^{2}\right\}<+\infty \quad, E\left\{\left\|[\mathbf{K}]^{-1}\right\|_{F}^{2}\right\}<+\infty \quad .
$$


We then have to construct a probability model for symmetric positive-definite real random matrices $[\mathbf{M}]$, $[\mathbf{D}]$ and $[\mathbf{K}]$ with the available information defined by Eqs. (49)-(50). This construction is performed using the results presented in Section 2.

\subsection{Nonparametric probability model of the reduced matrix model}

In this section we complete the construction of the probability model introduced in Section 3.3 using the developments of Section 2. Let $n_{0} \geq 1$ be a fixed integer and $n \geq n_{0}$. We apply the results of Section 2 to the set of positive-definite symmetric real random matrices $\{[\mathbf{M}],[\mathbf{D}],[\mathbf{K}]\}$ defined in Section 3.3, for which the available information is described by Eqs. (49)-(50). As indicated in Section 2.3, the levels of dispersion of random matrices $[\mathbf{M}],[\mathbf{D}]$ and $[\mathbf{K}]$ are controlled by parameters $\delta_{M}, \delta_{D}$ and $\delta_{K}$ respectively, which are independent of $n$ and are chosen such that (see Eq. (29)),

$$
0<\delta_{M}, \delta_{D}, \delta_{K}<\sqrt{\frac{n_{0}+1}{n_{0}+5}}
$$

Parameters $\lambda_{M}, \lambda_{D}$ and $\lambda_{K}$ are defined by Eq. (26),

$$
\lambda_{M}=\ell_{M}(n), \lambda_{D}=\ell_{D}(n), \lambda_{K}=\ell_{K}(n),
$$

in which $\ell_{M}(n), \ell_{D}(n)$ and $\ell_{K}(n)$ are given by Eq. (27),

$$
\begin{aligned}
& \ell_{M}(n)=\frac{1-\delta_{M}^{2}}{2 \delta_{M}^{2}} n+\frac{1+\delta_{M}^{2}}{2 \delta_{M}^{2}}, \\
& \ell_{D}(n)=\frac{1-\delta_{D}^{2}}{2 \delta_{D}^{2}} n+\frac{1+\delta_{D}^{2}}{2 \delta_{D}^{2}}, \\
& \ell_{K}(n)=\frac{1-\delta_{K}^{2}}{2 \delta_{K}^{2}} n+\frac{1+\delta_{K}^{2}}{2 \delta_{K}^{2}}
\end{aligned}
$$

From Section 2.6, we deduce that random matrices $[\mathbf{M}],[\mathbf{D}]$ and $[\mathbf{K}]$ are independent random variables with values in $\mathbf{M}_{n}^{+}(\mathbb{R})$ and their probability density functions $p_{[\mathbf{M}]}([M]), p_{[\mathbf{D}]}([D])$ and $p_{[\mathbf{K}]}([K])$ with respect to the measures (volume elements) $\widetilde{d} M, \widetilde{d} D$ and $\widetilde{d} K$ on $M_{n}^{S}(\mathbb{R})$ are given by Eqs. (11) and (13), and their characteristic functions by Eq. (12). 


\subsection{Construction of the stochastic transient response}

For fixed positive integer $n \geq n_{0}$, we have to construct stochastic processes $\left\{\mathbf{Y}_{n}(t), t \geq 0\right\}$ defined by Eqs. (46)-(48), stochastic process $\left\{R_{n}(t), t \geq 0\right\}$ defined (see Eq. (43)) by

$$
\begin{aligned}
R_{n}(t) & =<[\underline{\mathbb{K}}] \mathbf{Y}_{n}(t), \mathbf{Y}_{n}(t)>^{1 / 2}<[\underline{\mathbb{K}}] \underline{\mathbf{y}}_{S}, \underline{\mathbf{y}}_{S}>^{-1 / 2} \\
& =<[\underline{K}] \mathbf{Q}(t), \mathbf{Q}(t)>^{1 / 2}<[\underline{\mathbb{K}}] \underline{\mathbf{y}}_{S}, \underline{\mathbf{y}}_{S}>^{-1 / 2},
\end{aligned}
$$

and finally, random variable $B_{n}$ defined (see Eq. (44)) by

$$
B_{n}=\max _{t \geq 0} R_{n}(t)
$$

Below, we present a formulation which is adapted to Monte Carlo numerical simulation.

For given matrices $[M],[D],[K]$ in $\mathbb{M}_{n}^{+}(\mathbb{R})$ let $t \mapsto \mathbf{q}_{\mathbf{F}}, \mathbf{q}_{0}, \mathbf{q}_{1}(t ;[M],[D],[K])$ be the solution from $\mathbb{R}^{+}$ into $\mathbb{R}^{n}$ of the deterministic second-order differential equation

$$
[M] \ddot{\mathbf{q}}_{\mathbf{F}}, \mathbf{q}_{0}, \mathbf{q}_{1}(t)+[D] \dot{\mathbf{q}}_{\mathbf{F}}, \mathbf{q}_{0}, \mathbf{q}_{1}(t)+[K] \mathbf{q}_{\mathbf{F}}, \mathbf{q}_{0}, \mathbf{q}_{1}(t)=\mathbf{F}(t) \quad, \quad t \geq 0
$$

with the initial conditions

$$
\mathbf{q}_{\mathbf{F}}, \mathbf{q}_{0}, \mathbf{q}_{1}(0)=\mathbf{q}_{0} \quad, \quad \dot{\mathbf{q}}_{\mathrm{F}}, \mathbf{q}_{0}, \mathbf{q}_{1}(0)=\mathbf{q}_{1}
$$

We deduce that stochastic process $\{\mathbf{Q}(t), t \geq 0\}$ which is the solution of the stochastic dynamical problem defined by Eqs. (47)-(48), can be written as

$$
\mathbf{Q}(t)=\mathbf{q}_{\mathbf{F}}, \mathbf{q}_{0}, \mathbf{q}_{1}(t ;[\mathbf{M}],[\mathbf{D}],[\mathbf{K}])
$$

It should be noted that if $\mathbf{q}_{0}=\mathbf{q}_{1}=0$, then $\mathbf{Q}(t)$ can usually be written as

$$
\mathbf{Q}(t)=\int_{0}^{t}[h(t-\tau)] \mathbf{F}(\tau) d \tau
$$

in which $t \mapsto[h(t)]$ is the matrix-valued impulse response function of the linear filter associated with second-order differential Eq. (58). If $\mathbf{q} \mapsto[\theta(\mathbf{q})]$ is a mapping from $\mathbb{R}^{n}$ into the set $M_{\nu_{1}, \nu_{2}}(\mathbb{R})$ of all the $\left(\nu_{1} \times \nu_{2}\right)$ real matrices, we have

$$
\begin{aligned}
E\{[\theta(\mathbf{Q}(t))]\} & =\int_{\mathbb{M}_{n}^{+}(\mathbb{R})} \int_{\mathbb{M}_{n}^{+}(\mathbb{R})} \int_{\mathbb{M}_{n}^{+}(\mathbb{R})}\left[\theta \left(\mathbf{q}_{\mathbf{F}}, \mathbf{q}_{0}, \mathbf{q}_{1}\right.\right. \\
& \times p_{[\mathbf{M}]}([M]) \times p_{[\mathbf{D}]}([D]) \times p_{[\mathbf{K}]}([K]) \widetilde{d} M \widetilde{d} D \tilde{d} K
\end{aligned}
$$


For instance, $R_{n}(t)$ defined by Eq. (56) can be written as $R_{n}(t)=[\theta(\mathbf{Q}(t))]$ with $\nu_{1}=\nu_{2}=1$.

Calculation of the stochastic transient response of the dynamical system with random uncertainties requires the numerical construction of mapping $\left.t \mapsto \mathbf{q}_{\mathrm{F}}, \mathbf{q}_{0}, \mathbf{q}_{1}(t ;[M],[D],[K])\right)$ as the solution of the deterministic Eqs. (58)-(59). Since matrices $[M],[D]$ and $[K]$ are full matrices (not diagonal) as samplings of random matrices $[\mathbf{M}],[\mathbf{D}]$ and $[\mathbf{K}]$, any integral representation of $\mathbf{Q}(t)$ (for instance such as Eq. (61) when $\mathbf{q}_{0}=\mathbf{q}_{1}=0$ ) is not really efficient but second-order differential Eq. (58) is solved directly using an unconditionally stable implicit step-by-step integration method (such as the Newmark integration scheme [16]) with initial conditions defined by Eq. (59). In addition, we have to calculate multiple integrals in a higher dimension (see Eq. (62)) for which a well suited method consists in using a Monte Carlo calculation with or without variance reduction procedures [27-30]. This method is very efficient if there is a Monte Carlo simulation procedure for random matrices $[\mathbf{M}],[\mathbf{D}]$ and $[\mathbf{K}]$ which is the case of the method presented in Sections 2.4 and 2.5. It should be noted that for many applications, integer $n$ is sufficiently high that $\lambda_{M}, \lambda_{D}$ and $\lambda_{K}$ can be considered as positive integers without introducing any significant limitation in the model. Applying Eqs. (31)-(32) to random matrices $[\mathbf{M}],[\mathbf{D}]$ and $[\mathbf{K}]$ yields

$$
\begin{aligned}
{[\mathbf{M}] } & =\frac{1}{m_{M}(n)} \sum_{j=1}^{m_{M}(n)}\left(\left[\underline{L}_{M}\right]^{T} \mathbf{X}_{j}\right)\left(\left[\underline{L}_{M}\right]^{T} \mathbf{X}_{j}\right)^{T}, \\
{[\mathbf{D}] } & =\frac{1}{m_{D}(n)} \sum_{j=1}^{m_{D}(n)}\left(\left[\underline{L}_{D}\right]^{T} \mathbf{Y}_{j}\right)\left(\left[\underline{L}_{D}\right]^{T} \mathbf{Y}_{j}\right)^{T} \\
{[\mathbf{K}] } & =\frac{1}{m_{K}(n)} \sum_{j=1}^{m_{K}(n)}\left(\left[\underline{L}_{K}\right]^{T} \mathbf{Z}_{j}\right)\left(\left[\underline{L}_{K}\right]^{T} \mathbf{Z}_{j}\right)^{T}
\end{aligned}
$$

in which

$$
m_{M}(n)=\operatorname{Fix}\left(\frac{n+1}{\delta_{M}^{2}}\right) \quad, \quad m_{D}(n)=\operatorname{Fix}\left(\frac{n+1}{\delta_{D}^{2}}\right) \quad, \quad m_{K}(n)=\operatorname{Fix}\left(\frac{n+1}{\delta_{K}^{2}}\right),
$$

where $\operatorname{Fix}(x)$ is equal to $x$ when $x$ is an integer and $\operatorname{Fix}(x)$ rounds down $x+1$ to the nearest integer when $x$ is not an integer. In Eqs. (63)-(65), $\left[\underline{L}_{M}\right],\left[\underline{L}_{D}\right]$ and $\left[\underline{L}_{K}\right]$ are upper triangular matrices in $M_{n}(\mathbb{R})$ corresponding to the Cholesky factorization of symmetric positive-definite matrices $[\underline{M}],[\underline{D}]$ and $[\underline{K}]$ :

$$
[\underline{M}]=\left[\underline{L}_{M}\right]^{T}\left[\underline{L}_{M}\right] \quad, \quad[\underline{D}]=\left[\underline{L}_{D}\right]^{T}\left[\underline{L}_{D}\right] \quad, \quad[\underline{K}]=\left[\underline{L}_{K}\right]^{T}\left[\underline{L}_{K}\right] .
$$

The set of all the components of vectors $\mathbf{X}_{1}, \ldots, \mathbf{X}_{m_{M}(n)}, \mathbf{Y}_{1}, \ldots, \mathbf{Y}_{m_{D}(n)}$ and $\mathbf{Z}_{1}, \ldots, \mathbf{Z}_{m_{K}(n)}$ with values in $\mathbb{R}^{n}$ is constituted of $m_{M}(n) \times n+m_{D}(n) \times n+m_{K}(n) \times n$ independent random variables, each of which is a real-valued second-order normalized Gaussian random variable (zero mean value and unit variance). 


\section{Simple example}

The mean structure is constituted of a rectangular homogeneous and isotropic plate located in the plane $(O x, O y)$ of a Cartesian coordinate system $(O x y z)$, in bending mode (the outplane displacement is $z$ ), with constant thickness $4 \times 10^{-4} \mathrm{~m}$, width $0.40 \mathrm{~m}$, length $0.50 \mathrm{~m}$, mass density $7800 \mathrm{~kg} / \mathrm{m}^{3}$, Young's modulus $2.1 \times 10^{11} \mathrm{n} / \mathrm{m}^{2}$ and Poisson ratio 0.29 . This plate is simply supported on 3 edges and free on the fourth edge corresponding to $y=0$ (see Figure 1). The mean finite element model is constituted of a regular rectangular mesh with a constant step size of $0.01 \mathrm{~m}$ in $x$ and $y$ (41 nodes in the width, 51 nodes in the length). Consequently, all the finite elements are the same and each one is a 4-nodes square plate element. There are 2000 finite elements and $m=6009$ degrees of freedom ( $z$-translations and $x$ and $y$-rotations). The eigenfrequencies $\underline{\omega}_{\alpha}=2 \pi \underline{f}_{\alpha}$ of the mean finite element model are $\underline{f}_{1}=1.94$, $\underline{f}_{2}=10.28, \underline{f}_{3}=15.47, \ldots, \underline{f}_{25}=167.44, \ldots, \underline{f}_{80}=527.29 \mathrm{~Hz}$. The initial conditions defined by Eq. (38) are such that $\mathbf{y}_{0}=\mathbf{y}_{1}=0$. External impulsive load vector $\mathbf{x}(t)$ defined in Section 3.1 is written as $\mathbf{x}(t)=p(t) \mathbf{g}$. Spatial part $\mathbf{g}=\left(g_{1}, \ldots, g_{m}\right) \in \mathbb{R}^{m}$ is independent of time $t$ and is such that $g_{j}=0$ for all $j$ in $\{1, \ldots, m\}$ except for the nine DOFs corresponding to the nodes whose $(x, y)$ coordinates are $(0.30,0.25),(0.30,0.26),(0.30,0.27),(0.31,0.25),(0.31,0.26),(0.31,0.27),(0.32,0.25),(0.32,0.26)$ and $(0.32,0.27)$, for which $g_{j}=1$ (see Figure 1). Impulse function $t \mapsto p(t)$ is the rectangular impulse function defined by $p(t)=p_{\max } \mathbb{1}_{\left[0, t_{1}\right]}(t)$ with $p_{\max }=1$ and $t_{1}=0.4 \mathrm{~s}$, in which $\mathbb{1}_{\left[0, t_{1}\right]}(t)=1$ if $t \in\left[0, t_{1}\right]$ and $\mathbb{1}_{\left[0, t_{1}\right]}(t)=0$ if $t \notin\left[0, t_{1}\right]$. Figure 2 shows the graph of impulse function $t \mapsto p(t)$ anf Figure 3 shows the graph of the modulus of its Fourier transform. It can be seen in Figure 3 that the main part of the energy of impulse function $p$ is distributed over the $[-15,15] \mathrm{Hz}$ frequency band in which there are 3 structural modes of the mean model. Consequently, only the first structural modes significantly contribute in the dynamical response and the structural modes whose eigenfrequencies are greater that $168 \mathrm{~Hz}(n>25)$ contribute mainly in the quasi-static response and not in the dynamical response. The mean damping matrix is $[\underline{\mathbb{D}}]=2 \xi \Omega_{\mathrm{ref}}[\underline{\mathbb{M}}]$ with $\Omega_{\mathrm{ref}}=2 \pi \times 10$ and $\xi=0.001$. The transient response of the mean finite element model is calculated by solving the time evolution problem defined by Eqs. (37)-(38) with $\underline{\mathbf{y}}(0)=\underline{\mathbf{y}}(0)=0$ using an unconditionaly stable implicit step-by-step integration method (Newmark integration scheme) with a time-step size $\Delta t=1 / 1300 \mathrm{~s}$. The maximum $0.5<[\underline{K}] \underline{\mathbf{y}}_{S}, \underline{\mathbf{y}}_{S}>$ of the quasi-static elastic energy of the mean finite element model is equal to 2.552 . The dynamic magnification factor $\underline{b}_{m}=\max _{t \geq 0} \underline{r}_{m}(t)$, in which $\underline{r}_{m}(t)$ is calculated by Eq. (45), is equal to 1.595. For fixed positive integer $n$, the mean reduced matrix problem defined by Eqs. (40)-(41) with $\underline{\mathbf{q}}(0)=\underline{\dot{\mathbf{q}}}(0)=0$ is solved with the same Newmark integration scheme with the same time step size 
$\Delta t=1 / 1300 \mathrm{~s}$. Figure 4 shows the convergence of the dynamic magnification factor $\underline{b}_{n}$ of the mean model, defined by Eq. (44), as dimension $n$ of the mean reduced matrix model increases. From Figure 4 , it can be deduced that the transient response of the mean model is reasonably converged when $n=80$ for which $\underline{b}_{n}=1.487$. Figure 5 shows the graph of function $t \mapsto \underline{r}_{n}(t)$ for $n=80$.

Concerning the structure with random uncertainties, we choose $n_{0}=1$ which allows the convergence analysis with respect to dimension $n$ of the reduced matrix model with random uncertainties to be performed for $n \geq n_{0}=1$. The dispersions of the generalized mass, damping and stiffness random matrices of the reduced matrix model with random uncertainties, are controlled by parameters $\delta_{M}, \delta_{D}$ and $\delta_{K}$ introduced in Section 3.4, which have to verify the constraints defined by Eq. (51),

$$
0<\delta_{M}, \delta_{D}, \delta_{K}<0.57
$$

The numerical simulations presented below correspond to the values

$$
\delta_{M}=0.1 \quad, \quad \delta_{D}=0.1 \quad, \quad \delta_{K}=0.1 \quad,
$$

which verify Eq. (68). We are interested in the random response ratio $R_{n}(t)$ defined by Eq. (56) and the random dynamic magnification factor $B_{n}$ defined by Eq. (57). The transient response of the structure with random uncertainties is calculated using the Monte Carlo numerical simulation method. For given generalized mass, damping and stiffness matrices, the time evolution problem defined by Eqs. (58)-(59) is solved with the same Newmark integration scheme and with the same time step size $\Delta t=1 / 1300 \mathrm{~s}$. The Monte Carlo numerical simulation is carried out with $n_{S}=1000$ samples, denoted as $\theta_{1}, \ldots, \theta_{n_{S}}$, for which the samples $t \mapsto R_{n}\left(t ; \theta_{1}\right), \ldots, t \mapsto R_{n}\left(t ; \theta_{n_{S}}\right)$ are numerically calculated. For $t$ fixed, the mean value of random variable $R_{n}(t)$ is estimated by

$$
E\left\{R_{n}(t)\right\} \simeq \frac{1}{n_{S}} \sum_{j=1}^{n_{S}} R_{n}\left(t ; \theta_{j}\right)
$$

The samples of random variable $B_{n}$ are such that

$$
B_{n}\left(\theta_{j}\right)=\max _{t \geq 0} R_{n}\left(t ; \theta_{j}\right)
$$

The mean value of random variable $B_{n}$ is estimated by

$$
E\left\{B_{n}\right\} \simeq \frac{1}{n_{S}} \sum_{j=1}^{n_{S}} B_{n}\left(\theta_{j}\right)
$$


Finally, we introduce the function $t \mapsto R_{n, \max }(t ; \boldsymbol{\theta})$ and the real number $B_{n, \max }(\boldsymbol{\theta})$ defined by

$$
\begin{aligned}
R_{n, \max }(t ; \boldsymbol{\theta}) & =\max _{j=1, \ldots, n_{S}} R_{n}\left(t ; \theta_{j}\right), \\
B_{n, \max }(\boldsymbol{\theta}) & =\max _{j=1, \ldots, n_{S}} B_{n}\left(\theta_{j}\right),
\end{aligned}
$$

in which $\boldsymbol{\theta}=\left(\theta_{1}, \ldots, \theta_{n_{S}}\right)$. Figure 6 shows function $n \mapsto E\left\{B_{n}\right\}$ calculated by Eq. (72) and Figure 7 shows the function $n \mapsto B_{n, \max }(\boldsymbol{\theta})$ calculated by Eq. (74). Figures 6 and 7 show that a reasonable convergence is obtained for $n=80$ and for $n=80$, the value of $B_{n, \max }(\boldsymbol{\theta})$ is 1.676 . This value has to be compared to the value for the mean model which is 1.487 . Figure 8 is relative to $n=80$ and shows three curves: the lower irregular thin solid line corresponds to the graph of function $t \mapsto \underline{r}_{n}(t)$, the lower smooth thick solid line to the graph of function $t \mapsto E\left\{R_{n}(t)\right\}$ calculated by Eq. (70) and the upper irregular thin solid line to the graph of function $t \mapsto R_{n, \max }(t ; \boldsymbol{\theta})$ defined by Eq. (73).

\section{Conclusion}

We have presented a new approach allowing the random uncertainties to be modeled by a nonparametric model for prediction of transient responses to impulsive loads in linear structural dynamics. The information used does not require the description of the local parameters of the mechanical model. The probability model is deduced from the use of the entropy optimization principle whose available information is constituted of the fundamental algebraic properties related to the generalized mass, damping and stiffness matrices which have to be positive-definite symmetric matrices, and the knowledge of these matrices for the mean reduced matrix model. An explicit construction and representation of the probability model have been obtained and are very well suited to algebraic calculus and to Monte Carlo numerical simulation in order to compute the transient responses of structures submitted to impulsive loads. The numerical analysis related to the convergence of the stochastic solution with respect to the dimension of the random reduced matrix model has been carried out.

\section{References}

[ 1] Chen PC, Soroka WW. Multi-degree dynamic response of a system with statistical properties. Journal of Sound and Vibration 1973; 37(4):547-556.

[ 2] Soong TT. Random differential equations in science and engineering, New York: Academic Press, 1973.

[ 3] Haug EJ, Choi KK, Komkov V. Design sensitivity analysis of structural systems, San Diego: Academic Press, 1986. 
[ 4] Ibrahim RA. Structural dynamics with parameter uncertainties. Applied Mechanics Reviews 1987; 40(3):309-328.

[ 5] Shinozuka M. Structural response variability. ASCE Journal of Engineering Mechanics 1987; 113(6):825842.

[ 6] Spanos PD, Zeldin BA. Galerkin sampling method for stochastic mechanics problems. ASCE Journal of Engineering Mechanics 1994; 120(5):1091-1106.

[ 7] Lin YK, Cai GQ. Probabilistic structural dynamics, New York: McGraw-Hill, 1995.

[ 8] Vanmarcke E, Grigoriu M. Stochastic finite element analysis of simple beams. ASCE Journal of Engineering Mechanics 1983; 109(5):1203-1214.

[ 9] Shinozuka M, Deodatis G. Response variability of stochastic finite element systems. ASCE Journal of Engineering Mechanics 1988; 114(3):499-519.

[ 10] Spanos PD, Ghanem RG. Stochastic finite element expansion for random media. ASCE Journal of Engineering Mechanics 1989; 115(5):1035-1053.

[ 11] Ghanem RG, Spanos PD. Stochastic finite elements: a spectral approach, New York: Springer-Verlag, 1991.

[ 12] Kleiber M, Tran DH, Hien TD. The stochastic finite element method, Chichester: John Wiley and Sons, 1992.

[ 13] Ditlevsen O, Tarp-Johansen NJ. Choice of input fields in stochastic finite elements. Probabilistic Engineering Mechanics 1998; 14(1-2):63-72.

[ 14] Ohayon R, Soize C. Structural acoustics and vibration, San Diego: Academic Press, 1998.

[ 15] Clough RW, Penzien J. Dynamics of structures, New York: McGraw-Hill, 1975.

[ 16] Bathe KJ, Wilson EL. Numerical methods in finite element analysis, New York: Prentice Hall, 1976.

[ 17] Zienkiewicz OC, Taylor RL. 4. The finite element method, vol, 1. New York: McGraw-Hill, 1989 vol. 2, 1991.

[ 18] Argyris J, Mlejnek HP. Dynamics of structures, Amsterdam: North-Holland, 1991.

[ 19] Soize C. ISSN 1159-09747. A nonparametric model of random uncertainties in linear structural dynamics. Progress in Stochastic Structural Dynamics, 152. Marseille: Publication du LMA-CNRS, 1999. p. 109-138.

[ 20] Soize C. A nonparametric model of random uncertainties for reduced matrix models in structural dynamics. Probabilistic Engineering Mechanics 2000; 15(3):277-294.

[ 21] Mehta, ML. Random matrices, Revised and enlarged second edition, London: Academic Press, 1991. 
[ 22] Shannon CE. A mathematical theory of communication. Bell System Tech. J. 1948; 27:379-423 \& 623-659.

[ 23] Jaynes ET. Information theory and statistical mechanics. Physical Review 1957; 106(4):620-630 \& 108(2):171-190.

[ 24] Kapur JN, Kesavan HK. Entropy optimization principles with applications, San Diego: Academic Press, 1992.

[ 25] Anderson TW. Introduction to multivariate statistical analysis, Chichester: John Wiley and Sons, 1958.

[ 26] Soize C. Maximum entropy approach for modeling random uncertainties in transient elastodynamics. Journal of Acoustical Society of America 2001; 109(5):-.

[ 27] Cochran WG. Sampling techniques, New York: John Wiley and Sons, 1977.

[ 28] Rubinstein RY. Simulation and the Monte Carlo method, New York: John Wiley and Sons, 1981.

[ 29] Kalos MH, Whitlock PA. Monte Carlo methods, Volume 1: Basics, New York: John Wiley and Sons, 1986.

[ 30] Bratley P, Fox BL, Schrage EL. A guide to simulation, 2nd edition, New York: Springer-Verlag, 1987. 


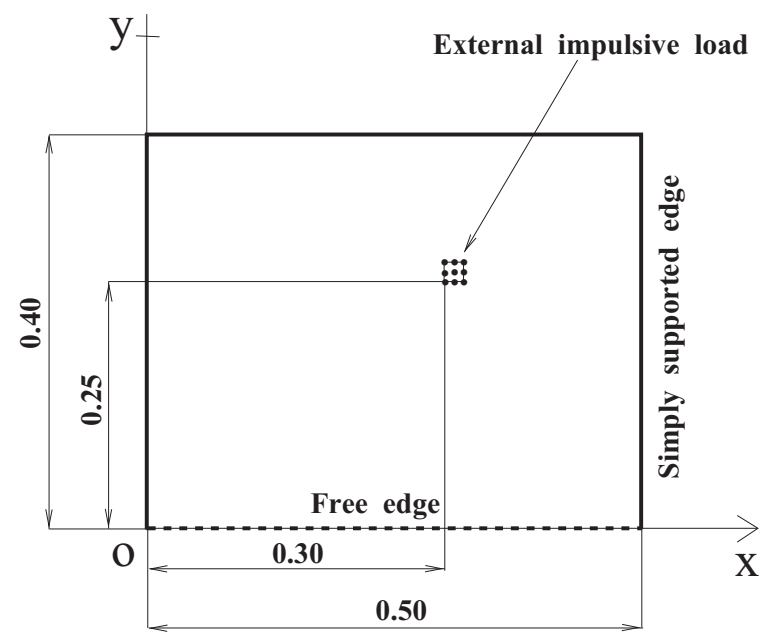

Figure 1. Geometry of the mean structure

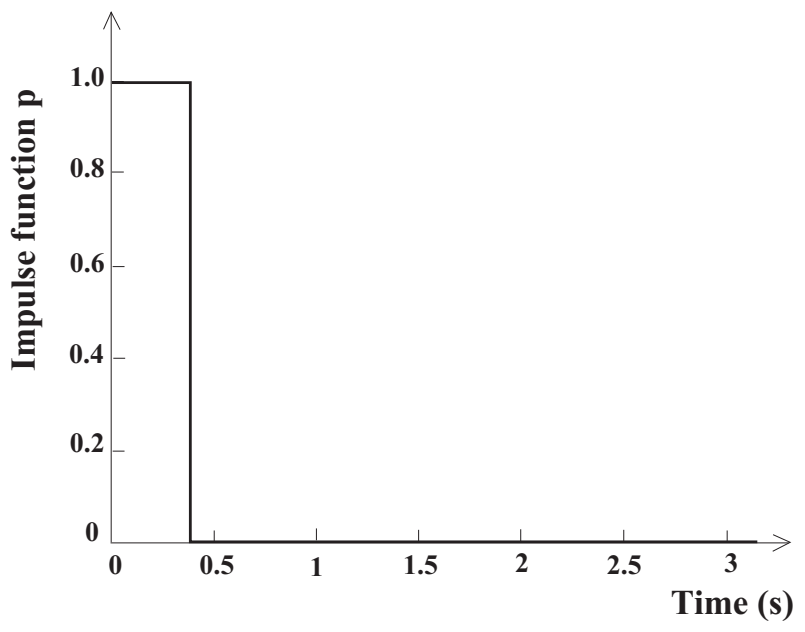

Figure 2. Graph of impulse function $t \mapsto p(t)$.

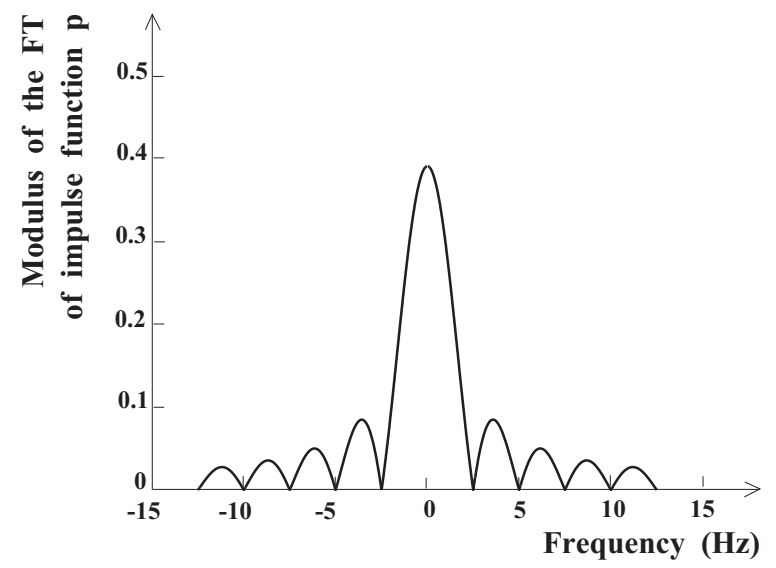

Figure 3. Graph of the modulus of the Fourier transform of impulse function.

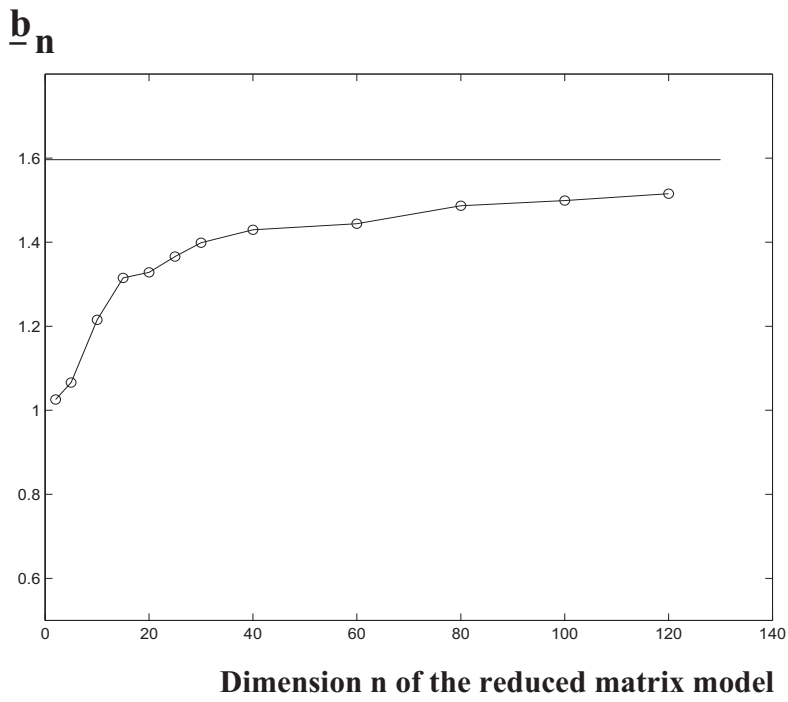

Figure 4. Graph of the convergence of dynamic magnification factor $\underline{b}_{n}$ with respect to dimension $n$ of the mean reduced matrix model.

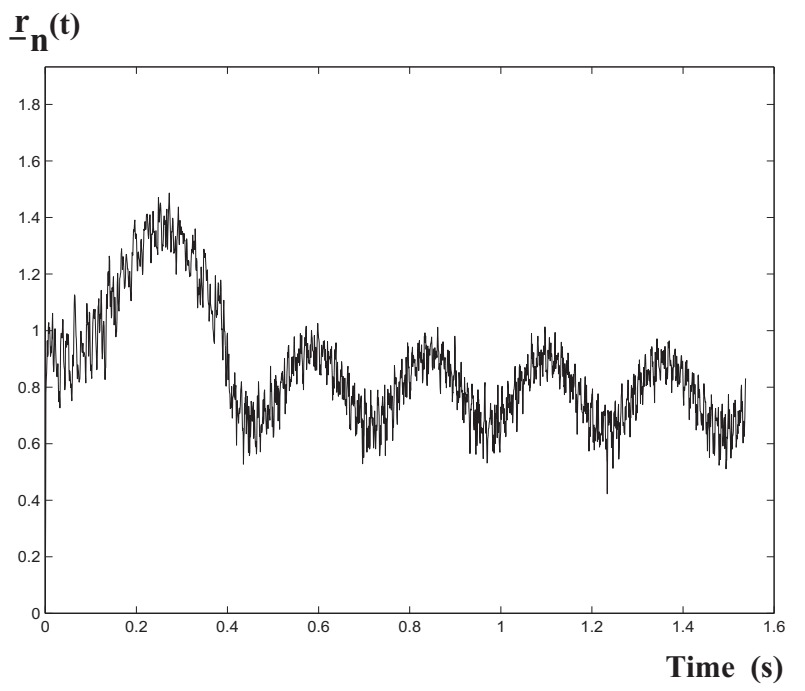

Figure 5. Graph of function $t \mapsto \underline{r}_{n}(t)$ for $n=80$ corresponding to the response ratio for the mean reduced matrix model. 


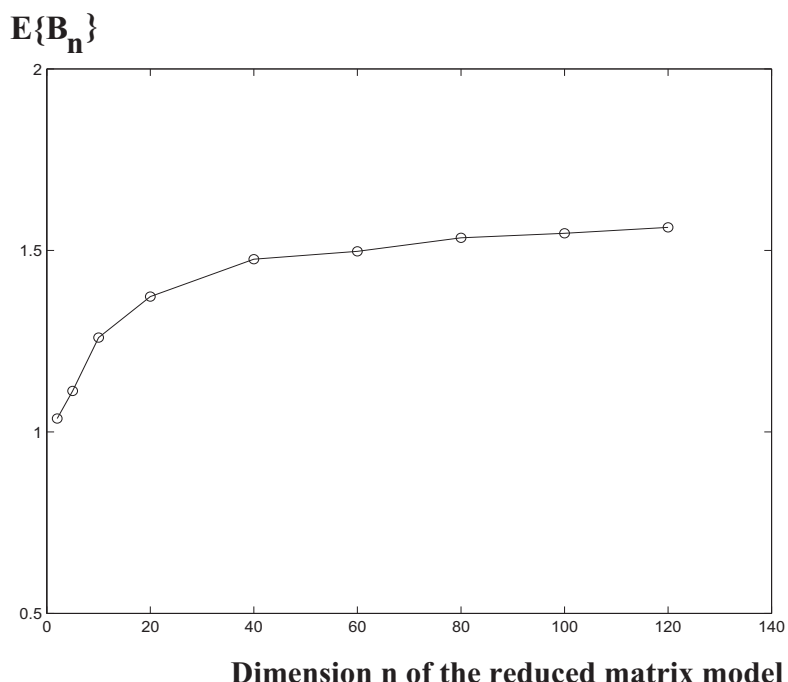

Figure 6. Graph of the convergence of $E\left\{B_{n}\right\}$ (mathematical expectation of the random dynamic magnification factor) with respect to dimension $n$ of the random reduced matrix model.

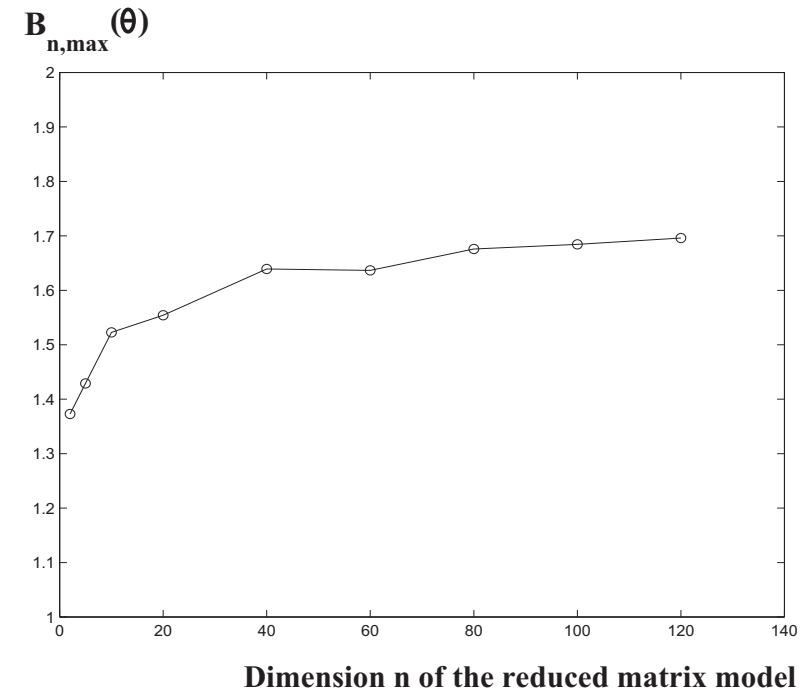

Figure 7. Graph of the convergence of $B_{n, \max }(\boldsymbol{\theta})$ (maximum of the random dynamic magnification factor) with respect to dimension $n$ of the random reduced matrix model.

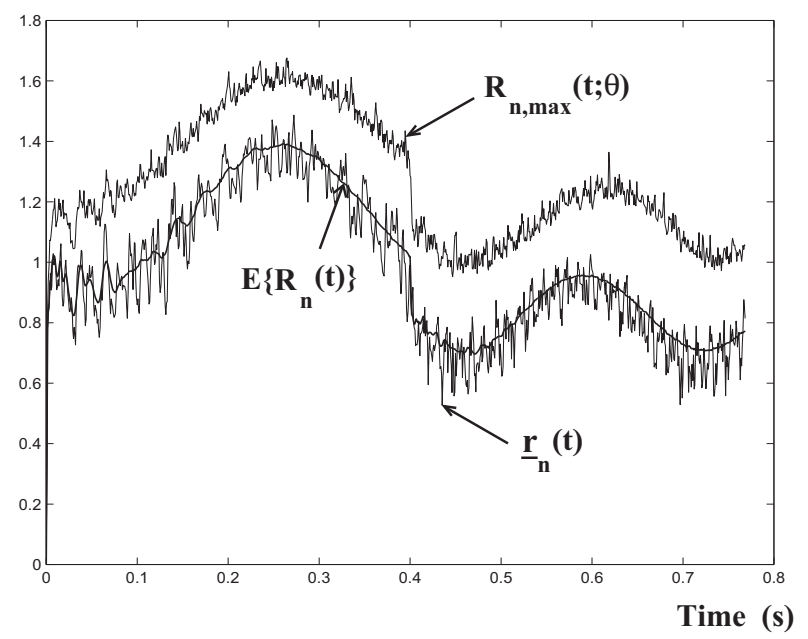

Figure 8. Transient responses $t \mapsto \underline{r}_{n}(t)$ (lower irregular thin solid line), $t \mapsto E\left\{R_{n}(t)\right\}$ (lower smooth thick solid line) and $t \mapsto R_{n, \max }(t ; \boldsymbol{\theta})$ (upper irregular thin solid line) for $n=80$ and $n_{S}=1000$. 